\title{
VANISHING CYCLES UNDER BASE CHANGE AND THE INTEGRAL HODGE CONJECTURE
}

\author{
MINGMIN SHEN
}

\begin{abstract}
In this paper we discuss an obstruction to the integral Hodge conjecture, which arises from certain behavior of vanishing cycles. This allows us to construct new counter-examples to the integral Hodge conjecture. One typical such counter-example is the product of a very general hypersurface of odd dimension and an Enriques surface. Our approach generalizes the degeneration argument of Benoist-Ottem [2].
\end{abstract}

\section{INTRODUCTION}

In this paper, we work over the field $\mathbb{C}$ of complex numbers. Let $X$ be a smooth projective variety, then the cohomology group of $X$ carries a Hodge structure given by

$$
\mathrm{H}^{k}(X, \mathbb{Z}) \otimes \mathbb{C}=\bigoplus_{p+q=k} \mathrm{H}^{p, q}(X), \quad \overline{\mathrm{H}^{p, q}(X)}=\mathrm{H}^{q, p}(X) .
$$

The group of integral Hodge classes, denoted $\operatorname{Hdg}^{2 p}(X, \mathbb{Z})$, consists of all the elements $\alpha \in \mathrm{H}^{2 p}(X, \mathbb{Z})$ such that $\alpha \otimes 1 \in \mathrm{H}^{2 p}(X, \mathbb{Z}) \otimes \mathbb{C}$ is in the summand $\mathrm{H}^{p, p}(X)$. One easily sees that the torsion classes are all integral Hodge classes, i.e.

$$
\mathrm{H}^{2 p}(X, \mathbb{Z})_{\text {tor }} \subseteq \operatorname{Hdg}^{2 p}(X, \mathbb{Z})
$$

W. Hodge discovered that the cohomology class $[Z]$ of an algebraic cycle $Z$ on $X$ is always an integral Hodge class.

Conjecture 1.1 (Integral Hodge Conjecture). Every integral Hodge class is the cohomology class of an algebraic cycle.

It is known since Atiyah-Hirzebruch [1] that the integral Hodge conjecture is false. Since then, many theories and techniques were developed to construct more counter-examples. In the recent paper [2], Benoist and Ottem used a degeneration argument to show that certain integral Hodge class is not algebraic. In this paper, we generalize their method to produce more counter-examples.

Our method is based on the following simple observation. Let $Y$ be a smooth projective variety and $U \subseteq Y$ a dense open subvariety. If $Z_{U}$ is an algebraic cycle on $U$, then it extends to an algebraic cycle $Z$ on $Y$ by taking the closure. However, a (locally finite) toplological cycle $z_{U}$ on $U$ does not necessarily extend to one on $Y$. The main reason is that the closure of $z_{U}$ might have a nontrivial boundary. Hence a cohomology class being algebraic imposes stronger extension property on the class. We make the following definition to make the discussion easier.

Definition 1.2. Let $\pi: \mathcal{X} \rightarrow B$ be a flat projective morphism between smooth complete varieties. Let $0 \in B$ be a closed point such that $X=\mathcal{X}_{0}:=\pi^{-1} 0$ is a smooth fiber. Let $\alpha \in \mathrm{H}^{k}(X, R)$ be a cohomology class with coefficients in a commutative ring $R$. We say that $\alpha$ is extendable if the following hold.

- There exists a smooth complete variety $\tilde{B}$ together with a generically finite morphism $\tilde{B} \rightarrow B$.

- For some resolution $\tilde{\mathcal{X}}$ of $\mathcal{X}^{\prime}:=\mathcal{X} \times_{B} \tilde{B}$ and some preimage $\tilde{0} \in \tilde{B}$ of 0 , we have $X=\tilde{\mathcal{X}}_{\tilde{0}}:=\tilde{\pi}^{-1}(\tilde{0})$ where $\tilde{\pi}: \tilde{\mathcal{X}} \rightarrow \tilde{B}$ is the morphism induced by $\pi$.

- There exists a cohomology class $\tilde{\alpha} \in \mathrm{H}^{k}(\tilde{\mathcal{X}}, R)$ such that $\alpha=\left.\tilde{\alpha}\right|_{X}$.

Date: January 23, 2019.

2010 Mathematics Subject Classification.

Key words and phrases. integral Hodge conjecture, vanishing cycles. 
MINGMIN SHEN

Remark 1.3. Assume that $0 \in B$ is a very general point. If $\alpha \in \mathrm{H}^{2 p}(X, \mathbb{Z})$ is the class of an algebraic cycle $Z$, then $\alpha$ is extendable. Indeed, one can identify $X=\pi^{-1} 0$ with the geometric generic fiber $\mathcal{X}_{\bar{\eta}}$. The algebraic cycle $Z$ can then be defined over a finite extension of $\eta_{B}$. Then a standard argument shows that there exists some generically finite morphism $\tilde{B} \rightarrow B$ and an algebraic cycle $\mathcal{Z}^{\prime}$ on $\mathcal{X}^{\prime}:=\mathcal{X} \times{ }_{B} \tilde{B}$ such that $\left[\mathcal{Z}_{\tilde{0}}^{\prime}\right]=\alpha$. The strict transform $\tilde{\mathcal{Z}}$ of $\mathcal{Z}^{\prime}$ in the resolution $\tilde{\mathcal{X}}$ sastisfies $\left[\tilde{\mathcal{Z}}_{\tilde{0}}\right]=\alpha$. Thus we can simply take $\tilde{\alpha}=[\tilde{\mathcal{Z}}]$.

This remark gives rise to the following non-algebraicity criterion.

(Non-algebraicity criterion) If an integral Hodge class $\alpha$ is not extendable, then this class is not algebraic on a very general fiber.

Our first main result is the following non-extendability of vanishing cycles on odd dimensional smooth hypersurfaces.

Theorem 1.4 (Theorem 3.2). Let $\mathcal{X} \longrightarrow B=\mathbb{P}^{1}$ be a Lefschetz pencil of smooth hypersurfaces of odd dimension $n$. Let $X=\pi^{-1} 0$ be a smooth fiber. Then every non-zero element $\alpha \in \mathrm{H}^{n}(X, R)$ is non-extendable, where $R$ is a nonzero commutative ring.

This non-extendability can be used to obstruct algebraicity as follows. For simplicity, we take $S$ to be an Enriques surface. Then $\mathrm{H}^{3}(S, \mathbb{Z})=\mathbb{Z} / 2 \mathbb{Z}$ with a generator $u$.

Corollary 1.5 (Corollary [3.3). Let $X \subset \mathbb{P}^{n+2}$ be a very general hypersurface of odd dimension $n$. For every element $\alpha \in \mathrm{H}^{n}(X, \mathbb{Z})$ which is not divisible by 2 , the torsion class $\alpha \otimes u \in \mathrm{H}^{n+3}(X \times S, \mathbb{Z})$ is not extendable (in a Lefschetz pencil) and hence not algebraic.

Remark 1.6. The proof of the corollary reduces to the non-extendability of the image $\bar{\alpha}$ of $\alpha$ in $\mathrm{H}^{n}(X, \mathbb{Z} / 2 \mathbb{Z})$; see section 3 . In $[2$, Benoist and Ottem considered the case where $X=E$ is a very general elliptic curve. Their method involves an element $\alpha \in \mathrm{H}^{1}(E, \mathbb{Z})$. Instead of considering the topological extendability of $\bar{\alpha} \in \mathrm{H}^{1}(E, \mathbb{Z} / 2 \mathbb{Z})$, they consider the degeneration of the double cover $E^{\prime} \longrightarrow E$ associated to $\bar{\alpha}$. The obstruction in the Benoist-Ottem example was given an interpretation via unramified cohomology by Colliot-Thélène [3]. It is interesting to see if a similiar interpretation exist for our generalisation.

The counter-examples to the integral Hodge conjecture obtained via the above corollary are all around the range of middle degree cohomology.

Our method also works when $X$ is a hyperplane section of a smooth projective variety $Y$. This more general case is treated in Theorem 3.4. Our result shows that there exist integral Hodge classes which are not extendable. Given the outstanding Hodge conjecture, it is natural to ask whether every rational Hodge class on a very general fiber is extendable.

Acknowledgement. A large part of the computations in Section 2 were carried out in the summer of 2018 when I was visiting University of Science and Technology of China. I thank Mao Sheng for the invitation. I also thank John Ottem for the interesting discussions related to this paper. This research was partially supported by NWO Innovational Research Incentives Scheme 016.Vidi.189.015.

\section{VANISHING CYCLES UNDER BLOW-UP}

2.1. An induction process. Let $r \geq 2$ be an integer and let $X_{r}$ be a complex analytic space with a unique singular point $P_{r}$. Assume that $P_{r}$ has an open neighborhood $U_{r}$ such that

$$
U_{r} \cong\left\{(t, \mathbf{z}) \in \mathbb{C} \times \mathbb{C}^{n+1}: t^{r}=z_{0}^{2}+\cdots+z_{n}^{2}\right\} .
$$

Let $D_{\epsilon}^{n+1}:=\left\{\mathbf{x} \in \mathbb{R}^{n+1}:|\mathbf{x}| \leq \epsilon\right\}$ be the closed disc. We have continuous maps

$$
\varphi_{r, a}: D_{\epsilon}^{n+1} \longrightarrow U_{r}, \quad \varphi_{r, a}(\mathbf{x})=\left(\xi_{r}^{a}|\mathbf{x}|^{2 / r}, \mathbf{x}\right)
$$

where $\xi_{r}=\exp \left(\frac{2 \pi i}{r}\right)$ and $a=0,1, \ldots, r-1$. Let $M^{\prime}$ be the blow-up of $M=\mathbb{C} \times \mathbb{C}^{n+1}$ at the point $P=(0, \mathbf{0})$. Let $U_{r}^{\prime} \subset M^{\prime}$ be the strict transform of $U_{r}$ and $\rho: X_{r}^{\prime} \rightarrow X_{r}$ be the resulting blow-up of $X_{r}$ at the point $P_{r}$. We write

$$
M \backslash\{(0, \mathbf{0})\}=V \cup V_{0} \cup \cdots \cup V_{n}, \quad V=\{t \neq 0\}, \quad V_{i}=\left\{z_{i} \neq 0\right\} .
$$


Then $M^{\prime}$ admits a corresponding open cover

$$
M^{\prime}=V^{\prime} \cup V_{0}^{\prime} \cup \cdots \cup V_{n}^{\prime}
$$

Here $V^{\prime} \cong \mathbb{C} \times \mathbb{C}^{n+1}$ and the map $V^{\prime} \rightarrow V \cup\{(0, \mathbf{0})\}$ is given by

$$
\left(t, w_{0}, \ldots, w_{n}\right) \mapsto\left(t, t w_{0}, \ldots, t w_{n}\right) .
$$

Similarly, we have $V_{i}^{\prime} \cong \mathbb{C} \times \mathbb{C}^{n+1}$ and the map $V_{i}^{\prime} \rightarrow V_{i} \cup\{(0, \mathbf{0})\}$ is given by

$$
\left(t, w_{0}, \ldots, w_{n}\right) \mapsto\left(t w_{i}, w_{0} w_{i}, \ldots, w_{i-1} w_{i}, w_{i}, w_{i+1} w_{i}, \ldots, w_{n} w_{i}\right) .
$$

The exceptional divisor $E$ of the blow-up $M^{\prime} \rightarrow M$ is isomorphic to $\mathbb{P}^{n+1}$ and the open cover

$$
E=\left(E \cap V^{\prime}\right) \cup \bigcup_{i=0}^{n}\left(E \cap V_{i}^{\prime}\right)
$$

is the standard affine cover associated to the homogeneous coordinates $\left[T: Z_{0}: \cdots: Z_{n}\right]$ of $\mathbb{P}^{n+1}$.

We have the following commutative diagram

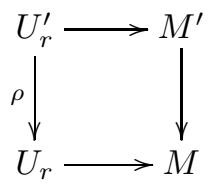

Furthermore, $U_{r}^{\prime} \cap V^{\prime} \subset V^{\prime}$ is defined by the equation

$$
t^{r-2}=w_{0}^{2}+\cdots+w_{n}^{2} .
$$

Thus $U_{r}^{\prime} \cap V^{\prime}$ is smooth if $r=2,3$; it is singular at the point $(t, \mathbf{w})=(0, \mathbf{0})$ if $r \geq 4$. The intersection $U_{r}^{\prime} \cap V_{i}^{\prime}$ is defined by the equation

$$
t^{r} w_{i}^{r-2}=w_{0}^{2}+\cdots+w_{i-1}^{2}+1+w_{i+1}^{2}+\cdots+w_{n}^{2}
$$

which is always smooth.

If $r=2$, then the exceptional divisor of $U_{r}^{\prime} \rightarrow U_{r}$ is the smooth quadric

$$
Q=\left\{T^{2}=Z_{0}^{2}+\cdots Z_{n}^{2}\right\} \subset E=\mathbb{P}^{n+1} .
$$

If $r \geq 3$, then the exceptional divisor of $U_{r}^{\prime} \rightarrow U_{r}$ is the singular quadric

$$
Q^{\prime}=\left\{0=Z_{0}^{2}+\cdots+Z_{n}^{2}\right\} \subset E=\mathbb{P}^{n+1} .
$$

The singular point of $Q^{\prime}$ is $P_{r}^{\prime}:=[1: 0: \cdots: 0] \in \mathbb{P}^{n+1}$.

The map $\varphi_{r, a}$ restricted to $D_{\epsilon}^{n+1} \backslash\{\mathbf{0}\}$ lifts to $V^{\prime}$, which is given by

$$
\mathbf{x}=\left(x_{0}, \ldots, x_{n}\right) \mapsto\left(\xi_{r}^{a}|\mathbf{x}|^{2 / r}, \xi_{r}^{-a}|\mathbf{x}|^{1-2 / r} \theta(\mathbf{x})\right), \quad \mathbf{x} \in D_{\epsilon}^{n+1} \backslash\{\mathbf{0}\},
$$

where $\theta(\mathbf{x})=\frac{\mathbf{x}}{|\mathbf{x}|} \in S^{n}$. If $r \geq 3$, then the above map extends to

$$
\varphi_{r, a}^{\prime}: D_{\epsilon}^{n+1} \longrightarrow V^{\prime}
$$

by the same formula and $\mathbf{0} \mapsto(0, \mathbf{0}) \in V^{\prime}$, which is the singular point of $Q^{\prime}$. In this case, $X_{r}^{\prime}$ is locally defined by the equation

$$
t^{r-2}=w_{0}^{2}+\cdots+w_{n}^{2} .
$$

The following lemma implies that the same argument can be repeated on $\left(X_{r-2}, P_{r-2}\right)=\left(X_{r}^{\prime}, P_{r}^{\prime}\right)$.

Lemma 2.1. (1) If $r=2$, then the lifting of $\left.\varphi_{2, a}\right|_{D_{\epsilon}^{n+1} \backslash\{\mathbf{0}\}}$ to $X_{r}^{\prime}$ can be extended to a continuous map

$$
\varphi_{2, a}^{\prime}:[0, \epsilon] \times S^{n} \longrightarrow X_{r}^{\prime}
$$

such that $\varphi_{2, a}^{\prime}(\rho, \mathbf{x})=\varphi_{2, a}(\rho \mathbf{x})$ for all $(\rho, \mathbf{x}) \in(0, \epsilon] \times S^{n}$. Furthermore,

$$
\varphi_{2, a}^{\prime}(0, \mathbf{x})=\left[1:(-1)^{a} x_{0}: \cdots:(-1)^{a} x_{n}\right] \in Q,
$$

which is an $n$-sphere in $Q \subset E \cong \mathbb{P}^{n+1}$ that vanishes in the homology of $\mathbb{P}^{n+1}$. In this case, $X_{r}^{\prime}$ is smooth. 
(2) If $r=3$, then the lifting of $\left.\varphi_{r, a}\right|_{D_{\epsilon}^{n+1} \backslash\{\mathbf{0}\}}$ to $X_{r}^{\prime}$ can be extended to a continuous map

$$
\varphi_{r, a}^{\prime}: D_{\epsilon}^{n+1} \longrightarrow X_{r}^{\prime}
$$

such that $\varphi_{r, a}^{\prime}(\mathbf{0})=P_{r}^{\prime}$ is the singular point of $Q^{\prime}$. In this case, $X_{r}^{\prime}$ is smooth.

(3) If $r \geq 4$, then $X_{r}^{\prime}$ is singular at the point $P_{r}^{\prime}$ where $X_{r}^{\prime}$ is locally defined by an equation

$$
t^{\prime r-2}={z^{\prime}}_{0}^{2}+z_{1}^{\prime 2}+\cdots+z_{n}^{\prime 2} \text {. }
$$

The lifting of $\left.\varphi_{r, a}\right|_{D_{\epsilon}^{n+1} \backslash\{\mathbf{0}\}}$ to $X_{r}^{\prime}$ can be extended to a continuous map

$$
\varphi_{r-2,0}: D_{\epsilon^{\prime}}^{n+1} \longrightarrow X_{r}^{\prime}
$$

with $\varphi_{r-2,0}(\mathbf{0})=P_{r}^{\prime}$ being the singular point of $X_{r}^{\prime}$ and $\varphi_{r-2,0}(\mathbf{x})=\left(|\mathbf{x}|^{\frac{2}{r-2}}, \mathbf{x}\right)$ for general $\mathbf{x}$.

Proof. For (1), we note that, in this case, the lifting of $\phi_{2, a}$ restricted to $D_{\epsilon}^{n+1} \backslash\{\mathbf{0}\}$ is given by

$$
\mathbf{x}=\left(x_{0}, \ldots, x_{n}\right) \mapsto\left((-1)^{a}|\mathbf{x}|,(-1)^{a} \theta(\mathbf{x})\right) .
$$

Note that $(0, \epsilon] \times S^{n} \cong D_{\epsilon}^{n+1} \backslash\{\mathbf{0}\}$. It is clear that the above map extends to a continuous map $\varphi_{2, a}^{\prime}$ : $[0, \epsilon] \times S^{n}$ as stated.

Statement (2) can be shown similarly.

We show the last statement and assume that $r \geq 4$. We have already seen that $X_{r}^{\prime}$ has a unique singular point $P_{r}^{\prime}$ such that $X_{r}^{\prime}$ is locally defined by

$$
t^{r-2}=w_{0}^{2}+w_{1}^{2}+\cdots+w_{n}^{2}
$$

and that there is a lifting $\varphi_{r, a}^{\prime}$ of $\varphi_{r, a}$ given by

$$
\varphi_{r, a}^{\prime}(\mathbf{x})=\left(\xi_{r}^{a}|\mathbf{x}|^{2 / r}, \xi_{r}^{-a}|\mathbf{x}|^{1-2 / r} \theta(\mathbf{x})\right), \quad \mathbf{x} \in D_{\epsilon}^{n+1} \backslash\{\mathbf{0}\}
$$

and $\varphi_{r, a}^{\prime}(\mathbf{0})=(0, \mathbf{0})=P_{r}^{\prime}$. We introduce a new set of coordinates

$$
\begin{aligned}
t^{\prime} & =\xi_{r}^{-a} t, \\
z_{i}^{\prime} & =\xi_{r}^{a} w_{i}
\end{aligned}
$$

and we see that the local defining equation of $X_{r}^{\prime}$ around $P_{r}^{\prime}$ becomes

$$
t^{\prime r-2}=z_{0}^{\prime 2}+z_{1}^{\prime 2}+\cdots+z_{n}^{\prime 2} .
$$

Furthermore, in terms of $\left(t^{\prime}, \mathbf{z}^{\prime}\right)$, the map $\varphi_{r, a}^{\prime}$ becomes

$$
\mathbf{x} \mapsto\left(|\mathbf{x}|^{2 / r},|\mathbf{x}|^{1-2 / r} \theta(\mathbf{x})\right)
$$

for $\mathbf{x} \neq \mathbf{0}$ and $\mathbf{0} \mapsto(0, \mathbf{0})$. Let $\epsilon^{\prime}=\epsilon^{1-2 / r}$ and define a homeomorphism $D_{\epsilon}^{n+1} \rightarrow D_{\epsilon^{\prime}}^{n+1}$ by $\mathbf{x}^{\prime} \mapsto \mathbf{x}^{\prime}=$ $|\mathbf{x}|^{-2 / r} \mathbf{x}$ for $\mathbf{x} \neq \mathbf{0}$ and $\mathbf{0} \mapsto \mathbf{x}^{\prime}=\mathbf{0}$. It follows that the composition $D_{\epsilon^{\prime}}^{n+1} \rightarrow D_{\epsilon}^{n+1} \rightarrow X_{r}^{\prime}$ becomes

$$
\mathbf{x}^{\prime} \mapsto\left(\left|\mathbf{x}^{\prime}\right|^{\frac{2}{r-2}}, \mathbf{x}^{\prime}\right)
$$

This concludes the proof.

\subsection{Application to vanishing cycles.}

2.3. Local situation. Let $\Delta \subset \mathbb{C}$ be the unit open disc in the complex plane and $\Delta^{*}=\Delta \backslash\{0\}$. Let $\pi: X \rightarrow \Delta$ be a proper map of complex manifolds such that $X^{*} \rightarrow \Delta^{*}$ is smooth, where $X^{*}=\pi^{-1} \Delta^{*}$. We write $X_{t}:=\pi^{-1} t, t \in \Delta$. Assume that $X_{0}=\pi^{-1}(0)$ has one ordinary double point $P$ such that we have local coordinates $\left(z_{0}, \ldots, z_{n}\right)$ on an open neighborhood $U$ of $P$ and

$$
\pi\left(z_{0}, z_{1}, \ldots, z_{n}\right)=z_{0}^{2}+z_{1}^{2}+\cdots+z_{n}^{2} .
$$

Let $\psi_{r}: \Delta \rightarrow \Delta$ be the map $\psi_{r}(t)=t^{r}$ and $X_{r}:=\psi_{r}^{*} X$ be the base change of $X$, where $r \geq 2$. Namely, we have the following fiber product quare

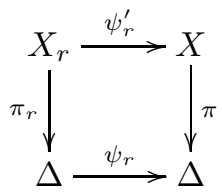


Let $U_{r}=\psi_{r}^{*} U$ be the corresponding base change of $U$. Thus $U_{r}$ is an open neighborhood of the point $P_{r}=\psi_{r}^{\prime-1}(P)$. Hence we have $U_{r} \subset \mathbb{C} \times \mathbb{C}^{n+1}$ defined be

$$
U_{r}=\left\{\left(t, z_{0}, \ldots, z_{n}\right) \in \mathbb{C} \times \mathbb{C}^{n+1}: t^{r}=z_{0}^{2}+z_{1}^{2}+\cdots+z_{n}^{2}\right\} .
$$

Then $P_{r}$ is the unique singular point of $U_{r}$ and it has coordinates $(0,0, \ldots, 0)$.

For any positive real number $\epsilon \in(0,1)$, let

$$
S_{\epsilon}^{n}=\left\{\left(\epsilon^{2}, x_{0}, \ldots, x_{n}\right) \in U_{r}: x_{0}^{2}+\cdots+x_{n}^{2}=\epsilon^{2}, x_{i} \in \mathbb{R}\right\} \subset X_{\epsilon^{2}}
$$

be a vanishing sphere. Let

$$
D_{\epsilon}^{n+1} \rightarrow X, \quad\left(x_{0}, \ldots, x_{n}\right) \mapsto\left(\sum x_{i}^{2}, x_{0}, \ldots, x_{n}\right)
$$

where $D_{\epsilon}^{n+1}=\left\{\left(x_{0}, \ldots, x_{n}\right) \in \mathbb{R}^{n+1}: x_{0}^{2}+\cdots+x_{n}^{2} \leq \epsilon^{2}\right\}$ is a small disc whose boundary gives the vanishing shpere $S_{\epsilon}^{n}$.

Let $\rho: X_{r}^{\prime} \rightarrow X_{r}$ be the blow-up of $X_{r}$ at the point $P_{r}$ and let $U_{r}^{\prime}:=\rho^{-1} U_{r}$. There are $r$ different ways to lift the map $D_{\epsilon}^{n+1} \backslash\{0\} \rightarrow X^{*}$ to $\left(X_{r}^{\prime}\right)^{*}$ given by

$$
\mathbf{x}=\left(x_{0}, \ldots, x_{n}\right) \mapsto\left(\xi_{r}^{a}|\mathbf{x}|^{2 / r}, x_{0}, \ldots, x_{n}\right), \quad a=0,1, \ldots, r-1 .
$$

We have seen in Lemma 2.1 that $X_{r}^{\prime}$ is again singular if $r \geq 4$ with a single singular point $P_{r}^{\prime}$ and the blow-up proess can be repeated.

Lemma 2.2. The following statements are true.

(1) The singularity of $X_{r}$ can be resolved by successively blowing up the singular points

$$
\tilde{X}=X_{r}^{(b)} \longrightarrow \cdots \longrightarrow X_{r}^{(2)} \longrightarrow X_{r}^{(1)}=X_{r}^{\prime} \longrightarrow X_{r}
$$

where $b=\left[\frac{r}{2}\right]$ and $X_{r}^{(b)}$ is smooth.

(2) Let $Q \subseteq \tilde{X}$ be the exceptional divisor of the last blow-up $X_{r}^{(b)} \longrightarrow X_{r}^{(b-1)}$. Then $Q$ is a component of $\tilde{X}_{0}=\tilde{\pi}^{-1}(0)$, where $\tilde{\pi}: \tilde{X} \longrightarrow \Delta$ is the composition of all the blow-ups together with $\pi_{r}$. If $r=2 b$ is even, then $Q$ is a smooth quardric hypersurface of dimension $n$; if $r=2 b+1$ is odd, then $Q$ is a cone over a smooth quadric hypersurface of dimension $n-1$.

(3) If $n$ is odd, then any of the $r$ liftings of the vanishing sphere $S_{\epsilon}^{n} \subset X_{\epsilon^{2}}$ to $\tilde{X}$ vanishes in $\mathrm{H}_{n}(\tilde{X}, \mathbb{Z})$.

(4) If $n$ is even and $r$ is odd, then any of the $r$ liftings of the vanishing sphere $S_{\epsilon}^{n} \subset X_{\epsilon^{2}}$ to $\tilde{X}$ vanishes in $\mathrm{H}_{n}(\tilde{X}, \mathbb{Z})$.

(5) If $n$ is even and $r$ is also even, then any of the $r$ the liftings of the vanishing sphere $S_{\epsilon}^{n} \subset X_{\epsilon^{2}}$ to $\tilde{X}$ is homologous to some sphere $S^{n} \subset Q$. Furthermore, the sphere $S^{n}$ vanishes in $\mathrm{H}_{n}\left(\mathbb{P}^{n+1}, \mathbb{Z}\right)$ under the embedding $Q \hookrightarrow \mathbb{P}^{n+1}$ of $Q$ as a quadric hypersurface.

Proof. Most of the statements are direct application of Lemma 2.1. We only need to prove (3) when $r$ is even. In this case, by (1) of Lemma 2.1, we know that the lifting of $S_{\epsilon}^{n} \subset X_{\epsilon^{2}}$ to $\tilde{X}$ is homologous to an $n$-sphere $S^{n} \subset Q$. Thus the homology class of the lifting of $S_{\epsilon}^{n}$ lands in the image of

$$
\mathrm{H}_{n}(Q, \mathbb{Z}) \longrightarrow \mathrm{H}_{n}(\tilde{X}, \mathbb{Z}) .
$$

When $n$ is odd, we have $\mathrm{H}_{n}(Q, \mathbb{Z})=0$ since a smooth quadric has trivial homology goup in odd degree. Thus we obtain the vanishing in (3).

2.4. Global situation. Let $\mathcal{X}$ be a smooth algeraic variety of dimension $n+1$ and $B$ a smooth curve. Let $\pi: \mathcal{X} \longrightarrow B$ be a proper morphism such that the following conditions holds.

- There exists a set $S=\left\{b_{1}, b_{2}, \ldots, b_{m}\right\} \subset B$ of finitely many points such that $X_{b_{i}}=\pi^{-1} b_{i}$ contains exactly one isolated singular point $P_{i}$ which is an ordinary double point.

- The morphism $\pi$ is smooth over $B \backslash S$.

Let $0 \in B$ be a point not in $S$ and let $X=X_{0}$. Thus $X$ is a smooth complete variety over $\mathbb{C}$. Let $\Delta_{i} \subset B$ be a small disc centered at $b_{i}$. Let $\Delta_{i}^{*}:=\Delta_{i} \backslash\left\{b_{i}\right\}$. 
Definition 2.3. A sphere $S_{\epsilon}^{n} \subset X_{t_{i}}, t_{i} \in \Delta_{i}^{*}$, is called a vanishing sphere associated to $P_{i}$ if the following conditions hold: (1) there exist local coordinates $\mathbf{z}=\left(z_{0}, z_{1}, \ldots, z_{n}\right)$ of $\mathcal{X}$ at $P_{i}=(0,0, \ldots, 0)$; (2) there is a local coordinate $t$ on $\Delta_{i}$ such that $\pi$ is locally given by

$$
t=\pi(\mathbf{z})=z_{0}^{2}+z_{1}^{2}+\cdots+z_{n}^{2}
$$

(3) with the above coordinates, we have $t_{i}=\epsilon^{2}$ and $S_{\epsilon}^{n}$ is given by all points $\mathbf{z}=\left(x_{0}, x_{1}, \ldots, x_{n}\right)$ with $x_{i} \in \mathbb{R}$ and $\sum x_{i}^{2}=\epsilon^{2}$.

Let $\gamma:[0,1] \longrightarrow B \backslash S$ be a continous path such that $\gamma(0)=0$ and $\gamma(1)=t_{i} \in \Delta_{i}^{*}$ for some $i$. Then

$$
\gamma_{*}: \mathrm{H}_{n}(X, \mathbb{Z}) \longrightarrow \mathrm{H}_{n}\left(X_{t_{i}}, \mathbb{Z}\right)
$$

is an isomorphism.

Definition 2.4. We say that a class $\alpha \in \mathrm{H}_{n}(X, \mathbb{Z})$ is a primitive vanishing class if there exists a path $\gamma:[0,1] \longrightarrow B \backslash S$ as above such that $\gamma_{*} \alpha \in \mathrm{H}_{n}\left(X_{t_{i}}, \mathbb{Z}\right)$ is the class of a vanishing sphere associated to $P_{i}$. A class $\alpha^{\prime} \in \mathrm{H}_{n}(X, \mathbb{Z})$ is a vanishing class (associated to $\mathcal{X} / B$ ) if it is an integral linear combination of primitive vanishing classes.

Proposition 2.5. Let $\mathcal{X}$ be a smooth algeraic variety and $B$ a smooth curve. Let $\pi: \mathcal{X} \longrightarrow B$ be a proper morphism as above. Let $0 \in B \backslash S$ and $X=X_{0}$. Let $\tilde{B}$ be another smooth curve and let $f: \tilde{B} \rightarrow B$ be a finite morphism. Let $\mathcal{X}^{\prime}:=\mathcal{X} \times_{B} \tilde{B}$ be the base change of $\mathcal{X}$ and let $\tilde{\mathcal{X}}$ be a resolution of $\mathcal{X}^{\prime}$. Let $\tilde{\pi}: \tilde{\mathcal{X}} \longrightarrow \tilde{B}$ be the resulting morphism induced from the morphism $\pi$. Let $\tilde{0} \in \tilde{B}$, such that $f(\tilde{0})=0$ and hence $X \cong \tilde{\pi}^{-1} \tilde{0}$. Let $j: X \hookrightarrow \tilde{\mathcal{X}}$ be the embedding. Let $\alpha \in \mathrm{H}_{n}(X, \mathbb{Z})$ be a vanishing class associated to $\mathcal{X} / B$.

(1) If $n$ is odd, then $j_{*} \alpha=0$ in $\mathrm{H}_{n}(\tilde{\mathcal{X}}, \mathbb{Z})$.

(2) If $n$ is even and $\tilde{\mathcal{X}}$ is obtained by successively blowing up the singular points, then $j_{*} \alpha$ is in the image of

$$
\bigoplus_{l=1}^{N} \mathrm{H}_{n}\left(Q_{l}, \mathbb{Z}\right)_{\text {van }} \longrightarrow \mathrm{H}_{n}(\tilde{\mathcal{X}}, \mathbb{Z}),
$$

where $Q_{l}$ runs through all smooth qudric hypersufaces appearing as components of the exceptional set of the morphism $\tilde{\mathcal{X}} \longrightarrow \mathcal{X}^{\prime}$ and $\mathrm{H}_{n}\left(Q_{l}, \mathbb{Z}\right)_{\text {van }}$ consists of classes $\beta \in \mathrm{H}_{n}\left(Q_{l}, \mathbb{Z}\right)$ that vanish in $\mathrm{H}_{n}\left(\mathbb{P}^{n+1}, \mathbb{Z}\right)$ under the natural embedding $Q_{l} \subset \mathbb{P}^{n+1}$.

Proof. We first look at the local behaviour of the morphism $f: \tilde{B} \longrightarrow B$ around a point $b_{i} \in S$. Assume that

$$
f^{-1} b_{i}=\left\{b_{i, 1}^{\prime}, b_{i, 2}^{\prime}, \ldots, b_{i, m_{i}}^{\prime}\right\} \subset \tilde{B} .
$$

For each point $b_{i, l}^{\prime}$, we can find a small disc $\Delta_{i, l} \subset \tilde{B}$ centered at $b_{i, l}^{\prime}$ such that the morphism $f: \tilde{B} \longrightarrow B$ restricts to the analytic map

$$
f_{i, l}: \Delta_{i, l} \longrightarrow \Delta_{i}, \quad z \mapsto z^{r_{i, l}} .
$$

To prove the proposition, we first assume that the resolution $\tilde{\mathcal{X}} \longrightarrow \mathcal{X}^{\prime}$ is the one obtained by successively blowing up the singular points. Without loss of generality, we may assume that $\alpha$ is a primitive vanishing class. Thus there is a path $\gamma:[0,1] \longrightarrow B \backslash S$ with $\gamma(0)=0 \in B$ and $\gamma(1)=t_{i} \in \Delta_{i}^{*}$ such that $\gamma_{*} \alpha$ is the class of a vanishing sphere in $X_{t_{i}}$. We may choose $\gamma$ in such a way that it avoids all the branching points of the morphism $f$. Thus there exists a unique lifting $\tilde{\gamma}:[0,1] \longrightarrow \tilde{B}$ such that $\tilde{\gamma}(0)=\tilde{0}$. Then $\tilde{\gamma}(1)=\tilde{t}_{i} \in \Delta_{i, l}^{*}$ for some $l \in\left\{1,2, \ldots, m_{i}\right\}$. Furthermore, $\tilde{\gamma}_{*} \alpha$ is the class of a lifting $\tilde{S}_{\epsilon}^{n}$ of the vanishing sphere $S_{\epsilon}^{n}$ in $X_{t_{i}}$. If $n$ is odd, then by Lemma 2.2 (3) we know that the homology class of $\tilde{S}_{\epsilon}^{n}$ vanishes in $\mathrm{H}_{n}\left(\tilde{\pi}^{-1} \Delta_{i, l}, \mathbb{Z}\right)$ and hence also in $\mathrm{H}_{n}(\tilde{\mathcal{X}}, \mathbb{Z})$. Similarly, if $n$ is even, we conclude from Lemma 2.2 (4) and (5).

Now assume $n$ is odd. We still need to establish the vanishing on an arbitrary resolotion $\tilde{\mathcal{X}}_{1}$ of $\mathcal{X}^{\prime}$. Let $\tilde{\mathcal{X}}$ be the resolution of $\mathcal{X}^{\prime}$ obtained by successively blowing up the singular points. Then we can find 
another resolution $\tilde{\mathcal{X}}_{2}$ which dominates both $\tilde{\mathcal{X}}$ and $\tilde{\mathcal{X}}_{1}$, namely we have a diagram

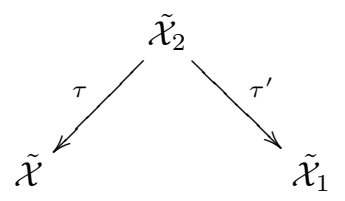

Let $j_{1}: X \hookrightarrow \tilde{\mathcal{X}}_{1}, j_{2}: X \hookrightarrow \tilde{\mathcal{X}}_{2}$ and $j: X \hookrightarrow \tilde{\mathcal{X}}$ be the inclusion of the fiber over $\tilde{0} \in \tilde{B}$ in the corresponding models. Set $\alpha_{1}=j_{1, *} \alpha, \alpha_{2}=j_{2, *} \alpha$ and $\tilde{\alpha}=j_{*} \alpha$ to be the corresponding homology classes. We have already see that $\tilde{\alpha}=0$. Since these models are isomorhic on an open neighborhood of the fiber $X$. We have $\alpha_{2}=\tau^{*} \tilde{\alpha}=0$ and $\alpha_{1}=\tau_{*}^{\prime} \alpha_{2}=0$.

\section{Applications to the integral Hodge conjecture}

In this section, we construct a class of new examples of the failure of the integral Hodge conjecture. These generalises the examples of Benoist-Ottem 2 .

Let $S$ be an Enriques surface. The cohomology groups of $S$ are described as follows.

$$
\begin{aligned}
\mathrm{H}^{0}(S, \mathbb{Z})=\mathbb{Z}, & \mathrm{H}^{0}(S, \mathbb{Z} / 2 \mathbb{Z})=\mathbb{Z} / 2 \mathbb{Z}, \\
\mathrm{H}^{1}(S, \mathbb{Z})=0, & \mathrm{H}^{1}(S, \mathbb{Z} / 2 \mathbb{Z})=\mathbb{Z} / 2 \mathbb{Z}, \\
\mathrm{H}^{2}(S, \mathbb{Z})=\mathbb{Z}^{\oplus 10} \oplus \mathbb{Z} / 2 \mathbb{Z}, & \mathrm{H}^{2}(S, \mathbb{Z} / 2 \mathbb{Z})=(\mathbb{Z} / 2 \mathbb{Z})^{\oplus 12}, \\
\mathrm{H}^{3}(S, \mathbb{Z})=\mathbb{Z} / 2 \mathbb{Z}, & \mathrm{H}^{3}(S, \mathbb{Z} / 2 \mathbb{Z})=\mathbb{Z} / 2 \mathbb{Z}, \\
\mathrm{H}^{4}(S, \mathbb{Z})=\mathbb{Z}, & \mathrm{H}^{4}(S, \mathbb{Z} / 2 \mathbb{Z})=\mathbb{Z} / 2 \mathbb{Z} .
\end{aligned}
$$

3.1. Special case: hypersurfaces. Let $X \subset \mathbb{P}^{n+1}$ be a smooth hypersurface. Assume that the dimension $n$ of $X$ is odd. By Lefschetz Hyperplane Theorem, we know that

$$
\mathrm{H}^{p}\left(\mathbb{P}^{n+1}, \mathbb{Z}\right) \longrightarrow \mathrm{H}^{p}(X, \mathbb{Z})
$$

is an isomorphism for $p<n$. Thus $\mathrm{H}^{<n}(X, \mathbb{Z})$ is torsion free and algebraic. Similarly, $\mathrm{H}_{<n}(X, \mathbb{Z})$ is torsion free. By Serre duality, we conclude that $\mathrm{H}^{>n}(X, \mathbb{Z})$ is also torsion free and so is $\mathrm{H}_{>n}(X, \mathbb{Z})$. Then by the universal coefficient thoerem for cohomology, we see that

$$
\mathrm{H}^{n}(X, \mathbb{Z}) \cong \operatorname{Hom}_{\mathbb{Z}}\left(\mathrm{H}_{n}(X, \mathbb{Z}), \mathbb{Z}\right) \oplus \mathrm{H}_{n-1}(X, \mathbb{Z})_{\text {tor }}
$$

is also torsion free. Hence we conclude that both $\mathrm{H}^{*}(X, \mathbb{Z})$ and $\mathrm{H}_{*}(X, \mathbb{Z})$ are torsion free.

Lemma 3.1. The following equality holds

$$
\mathrm{H}^{n+3}(X \times S, \mathbb{Z})=\bigoplus_{i=0}^{4} \mathrm{H}^{n+3-i}(X, \mathbb{Z}) \otimes \mathrm{H}^{i}(S, \mathbb{Z}) .
$$

Proof. The Künneth formula applied to this case gives

$$
\mathrm{H}^{n+3}(X \times S, \mathbb{Z})=\left(\bigoplus_{i=0}^{4} \mathrm{H}^{n+3-i}(X, \mathbb{Z}) \otimes \mathrm{H}^{i}(S, \mathbb{Z})\right) \oplus\left(\bigoplus_{i=0}^{4} \operatorname{Tor}_{1}\left(\mathrm{H}^{n+4-i}(X, \mathbb{Z}), \mathrm{H}^{i}(S, \mathbb{Z})\right)\right)
$$

Since the cohomology of $X$ is torsion free, we see that in the Tor ${ }_{1}$-term vanishes.

Theorem 3.2. Let $\pi: \mathcal{X} \longrightarrow B=\mathbb{P}^{1}$ be a Lefschetz pencil of smooth hypersurfaces of odd dimension $n$. Let $X=\pi^{-1} 0$ be a smooth fiber. Then any non-zero element $\alpha \in \mathrm{H}^{n}(X, R)$ is non-extendable, where $R$ is a non-zero commutative ring.

Proof. Assume that $\alpha$ is extendable. Then there exists a smooth projective curve $\tilde{B}$ and a finite morphism $\tilde{B} \longrightarrow B$ such that a resolution $\tilde{\mathcal{X}}$ of the base change $\mathcal{X}^{\prime}=\mathcal{X} \times_{B} \tilde{B}$ is obtained by successively blowing up the singular points. Let $\tilde{\pi}: \tilde{\mathcal{X}} \longrightarrow \tilde{B}$ be the induced morphism. Furthermore, we have a cohomology class $\tilde{\alpha} \in \mathrm{H}^{n}(\tilde{\mathcal{X}}, R)$ such that $\left.\tilde{\alpha}\right|_{X}=\alpha$, where $X=\tilde{\pi}^{-1} \tilde{0}$ for some preimage $\tilde{0} \in \tilde{B}$ of 0 . Let $j: X \hookrightarrow \tilde{\mathcal{X}}$ be the inclusion. Let $\beta \in \mathrm{H}_{n}(X, \mathbb{Z})$. Since $n$ is odd, we know that $\beta$ vahishes in $\mathrm{H}_{n}\left(\mathbb{P}^{n+1}, \mathbb{Z}\right)$. By 
Lefschetz theory (see for example 4]), we know that $\beta$ is a vanishing class associated to the Lefschetz pencil $\mathcal{X} \longrightarrow B$. Then by $(1)$ of Proposition 2.5, we see that $j_{*} \beta=0$ in $\mathrm{H}_{n}(\tilde{\mathcal{X}}, \mathbb{Z})$. Thus

$$
\langle\alpha, \beta\rangle=\left\langle j^{*} \tilde{\alpha}, \beta\right\rangle=\left\langle\tilde{\alpha}, j_{*} \beta\right\rangle=0 .
$$

This forces that $\alpha=0$ since $\mathrm{H}^{n}(X, R)=\operatorname{Hom}_{\mathbb{Z}}\left(\mathrm{H}_{n}(X, \mathbb{Z}), R\right)$.

Corollary 3.3. Let $i: X \hookrightarrow \mathbb{P}^{n+1}$ be a smooth hypersurface of odd dimension $n$ and let $S$ be an Enriques surface. Let $\alpha \in \mathrm{H}^{n}(X, \mathbb{Z})$ be an element not divisible by 2 and let $u \in \mathrm{H}^{3}(S, \mathbb{Z})$ be the unique nonzero element. Then the torsion class $\alpha \otimes u \in \mathrm{H}^{n+3}(X \times S, \mathbb{Z})$ is non-extendable in $\mathcal{X} \times S \longrightarrow B$ for any Lefschetz pencil $\mathcal{X} \longrightarrow B$ containing $X$. If $X$ is very genery, then $\alpha \otimes u$ is not algebraic.

Proof. Let $\pi: \mathcal{X} \longrightarrow B=\mathbb{P}^{1}$ be a Lefschetz pencil of hypersurfaces of dimension $n$ such that for some point $0 \in B$ the corresponding fiber $X_{0}:=\pi^{-1} 0 \cong X$. Assume that $\alpha \otimes u$ is extendable. As in the above proof, there exist a smooth projective curve $\tilde{B}$, a fninite morphism $\tilde{B} \rightarrow B$, a resolution $\tilde{\mathcal{X}}$ of $\mathcal{X}^{\prime}:=\mathcal{X} \times_{B} \tilde{B}$, an identification $X=\tilde{\pi}^{-1}(\tilde{0})$ and a cohomology class $\tau \in \mathrm{H}^{n+3}(\tilde{\mathcal{X}} \times S, \mathbb{Z})$ such that $\left.\tau\right|_{X \times S}=\alpha \otimes u$. Consider the class $\bar{\tau} \in \mathrm{H}^{n+3}(\tilde{\mathcal{X}} \times S, \mathbb{Z} / 2 \mathbb{Z})$ which is obtained from $\tau$ modulo 2 . Let $u^{\prime} \in \mathrm{H}^{1}(S, \mathbb{Z} / 2 \mathbb{Z})$ be the unique non-zero element which is associated to the $K 3$ covering $\tilde{S} \longrightarrow S$. Then we get

$$
\tilde{\alpha}:=\bar{\tau}^{*} u^{\prime} \in \mathrm{H}^{n}(\tilde{\mathcal{X}}, \mathbb{Z} / 2 \mathbb{Z})
$$

which stisfies the following condition

$$
\left.\tilde{\alpha}\right|_{X}=\left.\left(\bar{\tau}^{*} u^{\prime}\right)\right|_{X}=\left(\left.\bar{\tau}\right|_{X \times S}\right)^{*} u^{\prime}=(\bar{\alpha} \otimes \bar{u})^{*} u^{\prime}=\bar{\alpha}
$$

where $\bar{\alpha}$ is the image of $\alpha$ in $\mathrm{H}^{n}(X, \mathbb{Z} / 2 \mathbb{Z})$ and $\bar{u}$ is the image of $u$ in $\mathrm{H}^{3}(S, \mathbb{Z} / 2 \mathbb{Z})$. The last equality uses the duality relation $\left\langle\bar{u}, u^{\prime}\right\rangle=1$. It follows that $\bar{\alpha} \in \mathrm{H}^{n}(X, \mathbb{Z} / 2 \mathbb{Z})$ is extendable. By the above theorem, we have $\bar{\alpha}=0$ and hence $\alpha$ is divisible by 2 in $\mathrm{H}^{n}(X, \mathbb{Z})$. This give a contradiction.

It follows that $\alpha \otimes u$ is not algebraic for a very general memeber $X$ in a Lefschetz pencil. In particular, this holds for a very general $X$.

3.2. General case: hyperplane sections. Let $Y$ be a smooth projective variety with a very ample line bundle $\mathcal{O}_{Y}(1)$ which gives rise to an embedding $Y \hookrightarrow \mathbb{P}^{N}$. The same argument as above gives the following.

Theorem 3.4. Let $\pi: \mathcal{X} \rightarrow B=\mathbb{P}^{1}$ be a Lefschetz pencil in $\left|\mathcal{O}_{Y}(1)\right|$. Let $X=\pi^{-1} 0$ be a smooth fiber and let $i: X \hookrightarrow Y$ be the embedding. Assume that $\operatorname{dim} Y=n+1$ where $n$ is an odd integer. Let $R$ be a nonzero commutative ring.

(1) If $\alpha \in \mathrm{H}^{n}(X, R)$ is extendable, then we have

$$
\langle\alpha, \beta\rangle=0
$$

for all $\beta \in \mathrm{H}_{n}(X, \mathbb{Z})_{\text {van }}:=\operatorname{ker}\left\{i_{*}: \mathrm{H}_{n}(X, \mathbb{Z}) \longrightarrow \mathrm{H}_{n}(Y, \mathbb{Z})\right\}$. Furthermore, if $\mathrm{H}_{n}(Y, \mathbb{Z})$ vanishes and $\mathrm{H}_{n-1}(Y, \mathbb{Z})$ is torsion-free, then every nonzero element $\alpha \in \mathrm{H}^{n}(X, R)$ is non-extendable.

(2) Let $S$ be an Enriques surface and $u \in \mathrm{H}^{3}(S, \mathbb{Z})$ be the unique nonzero element. Let $\alpha \in \mathrm{H}^{n}(X, \mathbb{Z})$. If $\alpha \otimes u$, viewed as an element in $\mathrm{H}^{n+3}(X \times S, \mathbb{Z})$, is extendable (in the family $\mathcal{X} \times S \rightarrow B$ ), then $\bar{\alpha} \in \mathrm{H}^{n}(X, \mathbb{Z} / 2 \mathbb{Z})$ is extendable.

(3) Assume that $\mathrm{H}_{n}(Y, \mathbb{Z})$ vanishes and that $\mathrm{H}_{n-1}(Y, \mathbb{Z})$ is torsion-free. If $X$ is very general in $\left|\mathcal{O}_{Y}(1)\right|$, then for all $\alpha \in \mathrm{H}^{n}(X, \mathbb{Z})$ the class $\alpha \otimes u \in \mathrm{H}^{n+3}(X, \mathbb{Z})$ is not algebraic unless it is zero.

Proof. We will use the notations $\tilde{B}, \mathcal{X}^{\prime}, \tilde{\mathcal{X}}, j: X=\tilde{\pi}^{-1} \tilde{0} \hookrightarrow \tilde{\mathcal{X}}$ as in the previous proofs.

(1) If $\alpha$ is extendable, then there exists $\tilde{\alpha} \in \mathrm{H}^{n}(\tilde{\mathcal{X}}, R)$ such that $\alpha=j^{*} \tilde{\alpha}$. Then we again have

$$
\langle\alpha, \beta\rangle=\left\langle j^{*} \tilde{\alpha}, \beta\right\rangle=\left\langle\alpha, j_{*} \beta\right\rangle=0,
$$

since $j_{*} \beta=0$ for all $\beta \in \mathrm{H}_{n}(X, \mathbb{Z})_{\text {van }}$ by Lefschetz theory and (1) of Proposition 2.5. Assume that $\mathrm{H}_{n}(Y, \mathbb{Z})=0$, then we have

$$
\mathrm{H}^{n}(X, \mathbb{Z})_{\text {van }}=\mathrm{H}_{n}(X, \mathbb{Z}) .
$$

If $\mathrm{H}_{n-1}(Y, \mathbb{Z})$ is torsion free, then by Lefschetz hyperplane theorem, we know that $\mathrm{H}_{n-1}(X, \mathbb{Z})$ is also torsion free. Then the universal coefficient theorem for cohomology becomes

$$
\mathrm{H}^{n}(X, R)=\operatorname{Hom}_{\mathbb{Z}}\left(\mathrm{H}_{n}(X, \mathbb{Z}), R\right)
$$


Thus the vanishing of $\langle\alpha, \beta\rangle=0$ for all $\beta \in \mathrm{H}_{n}(X, \mathbb{Z})$ implies $\alpha=0$ in $\mathrm{H}^{n}(X, R)$.

(2) and (3): the proof is the same as that of Corollary 3.3. One only needs to note that ,under the assumptions of (3), the group $\mathrm{H}^{n+1}(X, \mathbb{Z})$ is also torsion free by Poincaré duality. Thus the universal coefficient theorem implies

$$
\mathrm{H}^{n}(X, \mathbb{Z} / 2 \mathbb{Z})=\mathrm{H}^{n}(X, \mathbb{Z}) \otimes \mathbb{Z} / 2 \mathbb{Z}
$$

Thus $\bar{\alpha}=0$ in $\mathrm{H}^{n}(X, \mathbb{Z} / 2 \mathbb{Z})$ if and only if $\alpha \otimes u=0$ in $\mathrm{H}^{n+3}(X \times S, \mathbb{Z})$ since the Künneth formula gives

$$
\left.\mathrm{H}^{n}(X, \mathbb{Z}) \otimes \mathrm{H}^{(} S, \mathbb{Z}\right) \hookrightarrow \mathrm{H}^{n+3}(X \times S, \mathbb{Z})
$$

and $\mathrm{H}^{3}(S, \mathbb{Z})=\mathbb{Z} / 2 \mathbb{Z}$. Then (3) follows from (1) and (2)

\section{REFERENCES}

[1] M. Atiyah and F. Hirzebruch, Analytic cycles on complex manifolds, Topology 1 (1962), p. 25-45.

[2] O. Benoist and J. Ottem, Failure of the integral Hodge conjecture for threefolds of Kodaira dimension zero, Commentarii Mathematici Helvetici, to appear.

[3] J.-L. Colliot-Thélène, Cohomologie non ramifiée dans le produit avec une courbe elliptique, preprint, 2018.

[4] K. Lamotke, The topology of complex projective varieties after S. Lefschetz, Topology 50 (1981), p. 15-51.

KdV Institute for Mathematics, University of Amsterdam, P.O.Box 94248, 1090 GE Amsterdam, Netherlands E-mail address: M.Shen@uva.nl 